\title{
EMG feedback tasks reduce reflexive stiffness during force and position perturbations
}

\author{
Patrick A. Forbes • Riender Happee • \\ Frans C. T. van der Helm • Alfred C. Schouten
}

Received: 6 May 2011/Accepted: 15 June 2011/Published online: 30 June 2011

(C) The Author(s) 2011. This article is published with open access at Springerlink.com

\begin{abstract}
Force and position perturbations are widely applied to identify muscular and reflexive contributions to posture maintenance of the arm. Both task instruction (force vs. position) and the inherently linked perturbation type (i.e., force perturbations-position task and position perturbations-force tasks) affect these contributions and their mutual balance. The goal of this study is to explore the modulation of muscular and reflexive contributions in shoulder muscles using EMG biofeedback. The EMG biofeedback provides a harmonized task instruction to facilitate the investigation of perturbation type effects irrespective of task instruction. External continuous force and position perturbations with a bandwidth of $0.5-20 \mathrm{~Hz}$ were applied at the hand while subjects maintained prescribed constant levels of muscular co-activation using visual feedback of an EMG biofeedback signal. Joint admittance and reflexive impedance were identified in the frequency domain, and parametric identification separated intrinsic muscular and reflexive feedback properties. In tests with EMG biofeedback, perturbation type (position and force) had no effect on joint admittance and reflexive impedance, indicating task as the dominant factor. A reduction in muscular and reflexive stiffness was observed when performing the EMG biofeedback task relative to the
\end{abstract}

\footnotetext{
P. A. Forbes $(\bowtie) \cdot$ R. Happee · F. C. T. van der Helm •

A. C. Schouten

Biomechanical Engineering, Department of Mechanical Engineering, Delft University of Technology,

Mekelweg 2, 2628 CD Delft, The Netherlands

e-mail: p.a.forbes@tudelft.nl

F. C. T. van der Helm - A. C. Schouten

Biomechanical Engineering Laboratory, Institute for Biomedical

Technology and Technical Medicine (MIRA),

University of Twente, 7500 EA Enschede, The Netherlands
}

position task. Reflexive position feedback was effectively suppressed during the equivalent EMG biofeedback task, while velocity and acceleration feedback were both decreased by approximately $37 \%$. This indicates that force perturbations with position tasks are a more effective paradigm to investigate complete dynamic motor control of the arm, while EMG tasks tend to reduce the reflexive contribution.

Keywords Biofeedback · Neuromuscular control · Electromyography · Afferent feedback .

Mechanical admittance $\cdot$ Reflexive impedance

\section{Introduction}

Posture maintenance of the arm is achieved using both muscular (intrinsic) and afferent (reflexive) contributions. Intrinsic muscle visco-elasticity increases with muscle activation levels (Agarwal and Gottlieb 1977), and coactivation (i.e., co-contraction) of muscles is an effective although energy consuming method of posture maintenance. Reflexive properties are determined by afferent feedback from sensory organs including muscle spindles (motion), Golgi tendon organs (force) and tactile receptors (touch and pressure). Reflex responses occur only after a perturbation, making reflexive feedback more energy efficient compared to co-activation. However, due to inherent neural time delays associated with afferent feedback, the effectiveness is limited (Schouten et al. 2008b). Under normal conditions, both mechanisms are balanced to maintain posture.

While the intrinsic muscular contribution to posture maintenance is well understood, the contribution of reflexes remains a continued focus of research. Experimental studies 
have shown that the reflexive contribution to posture maintenance depends on several factors, viz., muscle activation (Matthews 1986; Kirsch et al. 1993; Cathers et al. 2004), disturbance amplitude (Kearney and Hunter 1982; Sinkjaer et al. 1988; Stein and Kearney 1995; Cathers et al. 1999), the mechanical properties of the device with which the subject interacts (de Vlugt et al. 2002), task instruction (Akazawa et al. 1983; Doemges and Rack 1992; Dietz et al. 1994; Mugge et al. 2010) and the bandwidth of the perturbation signal (van der Helm et al. 2002; Schouten et al. 2008b; Mugge et al. 2010). Although these studies provide valuable insight into the afferent contribution to posture maintenance, the differences in experimental procedures make it difficult to compare results. In particular, the effect of perturbation type (force vs. position) is difficult to assess as it is inherently linked to the task instruction; position perturbations are used for force tasks and force perturbations are used for position tasks. The opposing nature of these task-perturbation combinations results in different reflexive behavior in both single (Akazawa et al. 1983; Doemges and Rack 1992) and multi-joint systems (Perreault et al. 2008).

The goal of this study is to explore the effect of perturbation type on reflexive feedback by using a harmonized task instruction across both position (PP) and force perturbations (FP). Any differences between the two would help identify either task or perturbation as the primary factor in the different observed reflex behaviors in the literature. Subjects performed electromyographical (EMG) biofeedback tasks (ET) representing desired co-contraction levels in the face of both perturbation types.

EMG biofeedback is a technique used for (re)learning of motor control in rehabilitation settings (Basmajian 1981; Holtermann et al. 2010). Subjects are provided with instantaneous feedback on the activity of measured motor units and instructed to perform various control tasks. Cathers et al. used EMG biofeedback as a task instruction to evaluate the effect of amplitude and bandwidth of stretch and voluntary muscle activation on the magnitude and timing of stretch reflexes in the flexor carpi radialis (Cathers et al. 1999, 2004). Subjects had to maintain specified levels of muscle activation while being exposed to position perturbations stretching the muscle. This flexion-only use of wrist muscles effectively made this task equivalent to a force task linked to a position perturbation. The task instruction proposed in the present study uses EMG biofeedback as a representation of co-contraction of antagonist shoulder muscles making this effectively a bi-directional stiffness task. Such an approach mediates the investigation of the effects of both muscle co-contraction level and perturbation type on muscular and reflex properties. Random continuous disturbances were applied to the arm, and system identification in the frequency domain was performed. Frequency response functions were estimated to capture the arm dynamics, and a neuromuscular control model was used to parameterize the physiological behavior in the form of intrinsic and reflexive components.

The effect of using EMG as a task instruction (ET) during force perturbations (FP) was first investigated by comparison to a position task (PT). Second, the effect of muscle (co-)activation level was investigated, a known influential factor on reflexes. Although this effect has been identified previously using position perturbations (i.e., force tasks), the effect has yet to be confirmed during force perturbations and under EMG biofeedback. Thirdly, the effect of perturbation type during this task was evaluated by applying a position perturbation to subjects which mimicked the position recorded during the force perturbations without informing the subjects that any change was made.

\section{Materials and methods}

Subjects

Ten healthy subjects (nine male) with an age range of 23-33 years having no self-reported history of neurological disorders or upper extremity injuries participated in these experiments. The experiment was in accordance with the Declaration of Helsinki, and all subjects gave informed consent prior to the experiment. All tests were performed on the right arm.

\section{Apparatus}

Subjects were seated in a comfortable upright posture, holding the handle of the manipulator (see Fig. 1) with an elbow flexion of $90^{\circ}$ (i.e., the reference position). The manipulator moved in forward and backward directions generating anteflexion and retroflexion of the glenohumeral joint (Wu et al. 2005). Details of the electro-hydraulic manipulator were described previously (Ruitenbeek and Janssen 1984; van der Helm et al. 2002). The manipulator could be position controlled or force controlled. During position control, the manipulator followed the prescribed motion regardless of the subject's resistance. During force control, the force measured between the handle and the actuator (i.e., the force imposed on the system by the subject) was used to generate the motion via a virtual massspring-damper system. In other words, the handle felt like a mass-spring-damper system to the subject. For this study, a virtual mass of $1 \mathrm{~kg}$, a damping of $0 \mathrm{~N} / \mathrm{ms}$ and a stiffness of $160 \mathrm{~N} / \mathrm{m}$ were used. Visual feedback was given on a 17-inch monitor mounted in front of the subject, where the signal presented matched the task. 


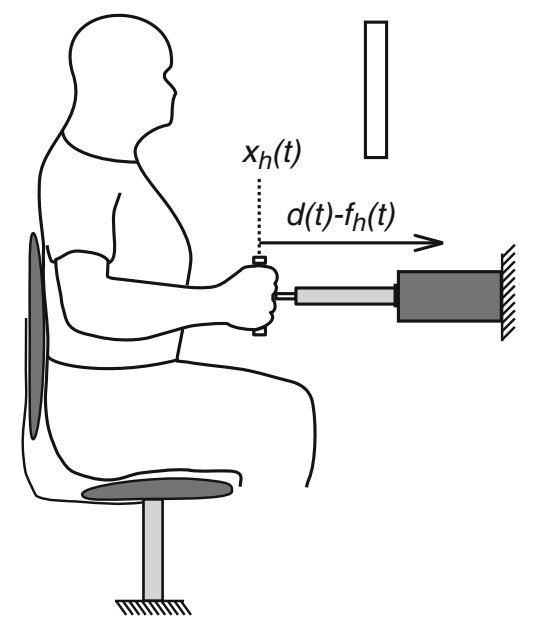

Fig. 1 Experimental setup showing a subject seated on a chair holding the handle with the right hand. The hand force, $f_{h}(t)$, applied to the manipulator is measured by a force transducer mounted between the handle and the piston. The manipulator controls the position of the handle, $x(t)$, based on the sum of the hand force, $f_{h}(t)$, external force disturbance, $d(t)$, and the simulated virtual dynamics (environment)

\section{Voluntary force experiments}

Isometric push-pull tests were used to determine the static EMG-to-force ratio, providing a relative measure of the muscle force, following previously described procedures (Schouten et al. 2008a). Visual feedback of the force was given to motivate and assist the subject. A maximum voluntary contraction test was performed first. Following a 5 -min break, subjects had to push or pull to fifteen different force levels $(0$ and $\pm 10,15,20,25,35,50,70 \mathrm{~N})$ and maintain the levels for $10 \mathrm{~s}$. These test series were conducted before and after the main experiments, and the average of the two was used for signal interpretation.

\section{Main experiments}

\section{Task instruction and test protocol}

The main experiment consists of multiple tasks, lasting $30 \mathrm{~s}$ each, while continuous force (FP) or position perturbations (PP) were applied. Additionally, tasks were performed with no perturbation (NP). The instructions associated with each task were as follows:

1. Position task (PT): subjects were instructed to "minimize displacement", while FP were applied. Visual feedback of the handle position was presented together with a horizontal line indicating the reference position.

2. EMG task (ET): subjects were instructed to match the displayed EMG co-activation signal (see "Analysis") to a target level which was displayed as a horizontal line. Four target levels were defined: 40, 70, 100 and $120 \%$ of the EMG co-activation signal recorded during PT. Three perturbations were applied during the performance of this task: FP, PP and NP.

3. Relax task (RT): subjects were instructed to "relax" as much as possible, while exposed to a FP. The monitor was switched off to remove visual feedback. This task was included to improve the estimate of the arm mass.

The experiment is summarized in Table 1. The PT and RT trials were conducted first in order to generate the reference co-activation levels required for all ET. The ET_FP trials were mixed randomly with half of the ET_NP trials and performed first. The displacement measured during the ET_FP trials was then used as the disturbance for the ET_PP trials (see "Perturbation signals"). These were performed mixed with the second half of the ET_NP trials in a random order. During ET trials, subjects were not informed on the type of disturbance (FP, PP or NP). Each test was performed four times resulting in 56 trials.

For all ET trials, subjects were instructed as a precondition to co-contract their muscles to the level required. The handle was locked prior to the start of the trial to prevent drift, and ET trials were started only when the absolute handle force was less than $3 \mathrm{~N}$ in order to preclude unidirectional muscle contraction strategies. The $160 \mathrm{~N} / \mathrm{m}$ stiffness included in the manipulator helped to prevent drift during the actual experiment. ET trials are described in the remainder of the article as ET_FP 40 , ET_FP ${ }_{70}, E_{-} \mathrm{FP}_{100}$ or ET_FP 120 , where PP replaces FP for position perturbation trials.

\section{Perturbation signals}

One perturbation signal was used as the disturbance in all FP trials with the exception of RT trials. The signal had a rectangular power spectrum with dominant power over a bandwidth of $0.5-1.5 \mathrm{~Hz}$ and was composed of multiple sinusoids, i.e., a multi-sine signal (Pintelon and Schoukens 2001). This was supplemented with a reduced power region according to methods developed by Mugge et al. (2007). The reduced power region $(1.5-20 \mathrm{~Hz})$ also had a rectangular spectrum and was designed to have $20 \%$ of the power in the dominant power region. Such an approach facilitates system identification over the whole bandwidth of $0.5-20 \mathrm{~Hz}$ while eliciting responses adapted to low bandwidth perturbations, which enhances reflexive contributions (van der Helm et al. 2002; Schouten et al. 2008b). Frequency averaging of four adjacent points was used for estimating the frequency response functions (see "Analysis"), resulting in only four clusters of four adjacent frequencies in the full-power region and 23 clusters in the reduced power region. The perturbation signal used in RT 
Table 1 Experimental matrix summarizing task instruction and perturbation characteristics

a $3.5 \mathrm{~mm}$ was the desired displacement

\begin{tabular}{|c|c|c|c|c|}
\hline \multirow[t]{2}{*}{ Condition } & \multirow[t]{2}{*}{ Task } & \multirow{2}{*}{$\begin{array}{l}\text { EMG co-act }(\%) \\
\text { (ET level) }\end{array}$} & \multicolumn{2}{|c|}{ Perturbation } \\
\hline & & & Type & RMS amp. (mm) \\
\hline PT_FP & Position & N/A & Force & $3.5^{\mathrm{a}}$ \\
\hline RT_FP & Relaxed & N/A & Force & 3.5 \\
\hline ET_FP & EMG & $40 / 70 / 100 / 120$ & Force & 3.5 \\
\hline ET_NP & EMG & $40 / 70 / 100 / 120$ & None & 0 \\
\hline ET_PP & EMG & $40 / 70 / 100 / 120$ & Position & 3.5 \\
\hline
\end{tabular}

trials had a bandwidth of $0.5-20 \mathrm{~Hz}$ consisting of 32 evenly distributed clusters with equal power.

PP signals were based on the measured average displacement of the four repeated FP trials at each specific ET level. In this way, the displacement for the position and force perturbations was equivalent at each ET level.

All signals were $30 \mathrm{~s}$ in length and had a sample frequency of 2,500 Hz. For analysis, approximately $4 \mathrm{~s}$ was removed from the beginning of all recorded data to avoid the inclusion of any transient effect. This resulted in a signal length of $2^{16}$ samples $(\sim 26.2 \mathrm{~s})$ and a frequency resolution of approximately $0.038 \mathrm{~Hz}$.

To compare the different conditions and to justify a linear modeling approach, the position deviations were kept within a consistent and limited range. For trials using FP, the magnitude of the force disturbance was scaled for each subject and each condition to ensure a root-meansquare (RMS) value for the position of approximately $3.5 \mathrm{~mm}$. This scaling was obtained prior to the actual experiments.

\section{Analysis}

\section{Data recording and processing}

During each trial, the handle position $x_{h}(t)$, handle force $f_{h}(t)$, disturbance force $d(t)$ and EMG were recorded at $2.5 \mathrm{kHz}$. The EMGs of four relevant shoulder muscles, two anteflexors ( $\mathrm{m}$. pectoralis major and $\mathrm{m}$. deltoideus anterior) and two retroflexors $(\mathrm{m}$. deltoideus posterior and $\mathrm{m}$. latissimus dorsi) were measured with differential surface electrodes (Delsys Bagnoli System, Delsys, Boston, USA). Before digitizing, the EMG signals were preamplified and bandpass filtered $(20-450 \mathrm{~Hz})$.

The EMG signals were used for two purposes: (1) realtime biofeedback of muscle co-activation (i.e., co-contraction) $\mathrm{c}_{\mathrm{a}}(t)$ during ET and (2) construction of a muscle activation signal $a(t)$ for system identification.

For biofeedback, the four individual signals were online high-pass filtered $(5 \mathrm{~Hz})$, rectified, normalized to their separate PT means and low-pass filtered at $0.3 \mathrm{~Hz}$ (secondorder Butterworth). The co-activation $\mathrm{c}_{\mathrm{a}}(t)$ was defined as the average of the four muscle signals. A cutoff of $0.3 \mathrm{~Hz}$ was chosen to minimize visible variations in the biofeedback signal due to reflexes evoked by the perturbations and thus preventing subjects from possibly reducing reflexive feedback. Lower cutoff frequencies resulted in slow responses of the biofeedback signal and poor task performance. The normalized activation signal of each muscle, as well as the paired anteflexor and retroflexor normalized activation signals, was also analyzed to assess task performance and task implementation.

The muscle activation signal $a(t)$ estimates the net amount of force generated using the EMG signals and EMGto-force ratios, and is described in detail in Schouten et al. (2008a). A brief description is provided in "Appendix 1".

\section{Non-parametric system identification}

The four repetitions of each test were averaged in time to reduce the effects of noise. The admittance and reflexive impedance frequency responses functions (FRF) were then estimated in the frequency domain using system identification techniques described in detail by van der Helm et al. (2002) and Schouten et al. (2008b). In general, FRFs describe the input-output relationship of a system as a function of frequency. At any frequency, the gain indicates the magnitude of the output relative to the input, and the phase indicates the timing of the output relative to the input. The admittance describes the displacement $\left(x_{h}\right)$ of the arm due to an input force $\left(f_{h}\right)$ and represents the inverse of the limb impedance. The reflexive impedance describes the muscle activation $(a)$ due to the handle displacement $\left(x_{h}\right)$ and reflects the afferent feedback. For FP trials, interaction between the subject and manipulator exists and closed loop algorithms were required to estimate the FRFs according to:

$\hat{H}_{f x}=\frac{\hat{S}_{d x}(f)}{\hat{S}_{d f}(f)}$

$\hat{H}_{x a}=\frac{\hat{S}_{d a}(f)}{\hat{S}_{d x}(f)}$

where $\hat{S}_{d x}(f)$ represents the estimated cross-spectral density between the disturbance $d$ and the handle position $x_{h}$. The cross-spectral densities are averaged across four adjacent 
frequencies to improve the estimate (Jenkins and Watts 1968). For all PP trials, standard open loop algorithms were used to estimate the FRFs, i.e., $d$ was replaced by $x$ in Eqs. 1-2.

The estimation of FRFs assumes linearity between the input and output signals, which was evaluated for both FP and PP by calculating the coherence according to:

$\hat{\gamma}_{d x}=\frac{\hat{S}_{d x}(f)^{2}}{\hat{S}_{d d}(f) \hat{S}_{x x}(f)}$

$\hat{\gamma}_{d a}=\frac{\hat{S}_{d a}(f)^{2}}{\hat{S}_{d d}(f) \hat{S}_{a a}(f)}$

where $d$ is replaced by $x$ for PP conditions. Coherence ranges from zero to one, where one reflects a perfectly linear noise free system. The FRFs and coherences were evaluated only at frequencies where the perturbation signal had power.

\section{Parameter estimation of neuromuscular model}

A neuromuscular model was employed to identify the physiological relevant parameters underlying the experiments (Schouten et al. 2008b; Mugge et al. 2010). The model facilitates the quantification of proprioceptive reflexes, muscle parameters and mass; see "Appendix 2" for details regarding the model along with a figure depicting the model structure. The muscle parameters include muscle stiffness and damping $(k$ and $b)$ as well as hand grip stiffness and damping $\left(k_{h}\right.$ and $\left.b_{h}\right)$. The proprioceptive reflex parameters include position, $k_{p}$, velocity, $k_{v}$, and acceleration, $k_{a}$, feedback gains as well as neural time delay, $t_{d}$. The acceleration term captures the observed second-order (acceleration) dynamics observed in the reflexive impedance, which is an artifact of the linearization of the unidirectional muscle spindle velocity, rather than a distinct sensory function (Kukreja et al. 2003; Stienen et al. 2007; Schouten et al. 2008b). Muscle activation dynamics were implemented as a second-order model using parameters obtained from previous studies (Schouten et al. 2008b).

Preliminary model fits included force feedback; however, the gain tended to approach zero for all conditions. In cases where $k_{f}$ was estimated to be other than zero, the results showed poor model validation and high parameter sensitivity. For these reasons, the contribution of force feedback was considered negligible or unquantifiable and was therefore excluded in the final estimations.

The parameters $\left(\tau_{d}, m, b, k, b_{h}, k_{h}, k_{p}, k_{v}, k_{a}\right)$ were identified by fitting the model on the estimated FRF of the mechanical admittance and reflexive impedance (Eqs. 1-2) simultaneously, using the criterion function below:

$$
\begin{aligned}
e= & \sum_{k} \frac{\hat{\gamma}_{d x}^{2}\left(f_{k}\right)}{1+f_{k}}\left|\log \left(\frac{\hat{H}_{f x}\left(f_{k}\right)}{H_{f x}\left(f_{k}\right)}\right)\right|^{2} \\
& +q \sum_{k} \frac{\hat{\gamma}_{x a}^{2}\left(f_{k}\right)}{1+f_{k}}\left|\log \left(\frac{\hat{H}_{x a}\left(f_{k}\right)}{H_{x a}\left(f_{k}\right)}\right)\right|^{2}
\end{aligned}
$$

where $f_{k}$ are the frequencies with signal power and $q$ is a weighting factor. A $q$ of 0.09 was chosen such that both terms have an approximately equal weighting.

The parameters were first estimated for the PT and RT results simultaneously, where the mass was equivalent across these two conditions and reflex gains were assumed to be zero in the RT condition. The values for arm mass and time delay were then fixed and used in estimating remaining intrinsic and reflexive parameters in ET conditions, where the ratio between intrinsic arm parameters, $b$ and $k$, was fixed compared to PT conditions. A similar assumption was made for the hand grip parameters $\left(b_{h}\right.$ and $\left.k_{h}\right)$.

\section{Model validation}

Validity of the estimated model parameters was assessed using the variance accounted for (VAF). VAF describes how much of the variance of the measured time signal is captured by the model, where $100 \%$ reflects a perfect relation. The estimated parameters were used to obtain simulated position $\hat{x}_{h}(t)$ and muscle activation $\hat{a}(t)$ responses using the input disturbance $d(t)$. Experimentally measured $x_{h}(t)$ and $a(t)$ were then used to calculate $\mathrm{VAF}_{x}$ and $\mathrm{VAF}_{a}$ according to:

$\mathrm{VAF}_{x}=1-\frac{\sum_{1}^{n}\left(x_{h}\left(t_{n}\right)-\hat{x}_{h}\left(t_{n}\right)\right)^{2}}{\sum_{1}^{n}\left(x_{h}\left(t_{n}\right)\right)^{2}}$

where for $\mathrm{VAF}_{a}, x_{h}$ and $\hat{x}_{h}$ are replaced by $a$ and $\hat{a}$, respectively, and $\mathrm{n}$ is the number of data points in the signal.

\section{Statistical analysis}

The effects of EMG level and perturbation type on the mean of the combined EMG co-activation signal $\left(c_{a}\right)$, the mean of the individual EMG signals and the estimated parameters were evaluated using a repeated-measures ANOVA. Similarly, these effects on the mechanical admittance and reflexive impedance were evaluated for significance on their gain and phase at each frequency point. In addition to the ANOVAs, a paired Student's $t$ test was used to evaluate the effect of task type (PT vs. ET_FP 100$)$. A significance of 0.05 was used for all analyses. 


\section{Results}

Task performance and implementation

Figure 2 presents a ten-second time segment of a typical subject's response during PT, ET_FP 100 and ET_PP 100 . Despite having similar displacement amplitude and coactivation, the handle force is smaller for both ET conditions, indicating an increased admittance (i.e., less stiff). The co-activation signal demonstrates the subject's ability to effectively perform the task for both perturbations, maintaining a prescribed level of muscle activity in the ET, which in these co-activation equivalent conditions matches the measured mean response during PT trials. Subjects were capable of maintaining relatively constant co-activation levels throughout all ET trials (FP, PP and NP). The variation (i.e., standard deviation) of the co-activation signal within the ET_FP 100 trials was only $30 \%$ of that measured in PT. No significant difference within the variation of the co-activation signal could be found when comparing perturbation types (i.e., FP vs. PP); while the presence of either perturbation resulted in a $4 \%$ increase in variation compared to NP $(P<0.05)$. Additionally, the variation of the co-activation signal increased with ET level $(P<0.05)$.

Figure 3 (top) plots the group averaged mean muscle activity signals during ET_FP trials of the combined anteflexors and retroflexors, as well as the total co-activation signal relative to the PT, at all ET levels. The results show an even distribution of anteflexor and retroflexor activity used to generate the co-activation signal across all subjects. A significant effect $(P<0.001)$ of ET level was seen in all muscular signals (both combined and individual) which increased with ET level. No significant effect could be attributed to perturbation type (FP, PP or NP). The low standard deviation of the co-activation signal indicated that all subjects performed the task effectively. The standard deviations of the anteflexors and retroflexors were larger, indicating that subjects could favor specific muscles to generate the co-activation signal.

Evaluation of individual subject responses revealed the presence of muscle favoring in most of the subjects ( 8 of 10). Figure 3 (bottom) plots the individual muscle responses from a subject with a preference toward the use of pectoralis major. For this subject, the favored muscle makes up consistently $\sim 40 \%$ of the total co-activation signal. Consistent favoring was also observed across perturbation types. Interestingly, the occurrence of muscle favoring did not result in position drift throughout FP trials.

Non-parametric frequency response functions

Figure 4 presents the mechanical admittance and reflexive impedance FRFs of a typical subject during PT and ET trials when subjected to FP. The mechanical admittance of the shoulder joint resembles a second-order system, i.e., a mass-spring-damper system. The low-frequency response (flat gain and no phase lag) is spring-like due to intrinsic and reflexive properties. The high-frequency response (i.e.,
Fig. 2 Time domain results of a typical subject during PT (thick grey), ET_FP 100 (solid black) and ET_PP 100 (dashed black), showing a the measured force $f_{h}$, b the measured position $x$ and $\mathbf{c}$ the co-activation signal $c_{a}$. The measured force during ET_FP 100 was almost identical and is difficult to differentiate in the figure. Also, the measured position during ET_FP trials was used as an input for ET_PP trials and was therefore not plotted for ET_PP 100
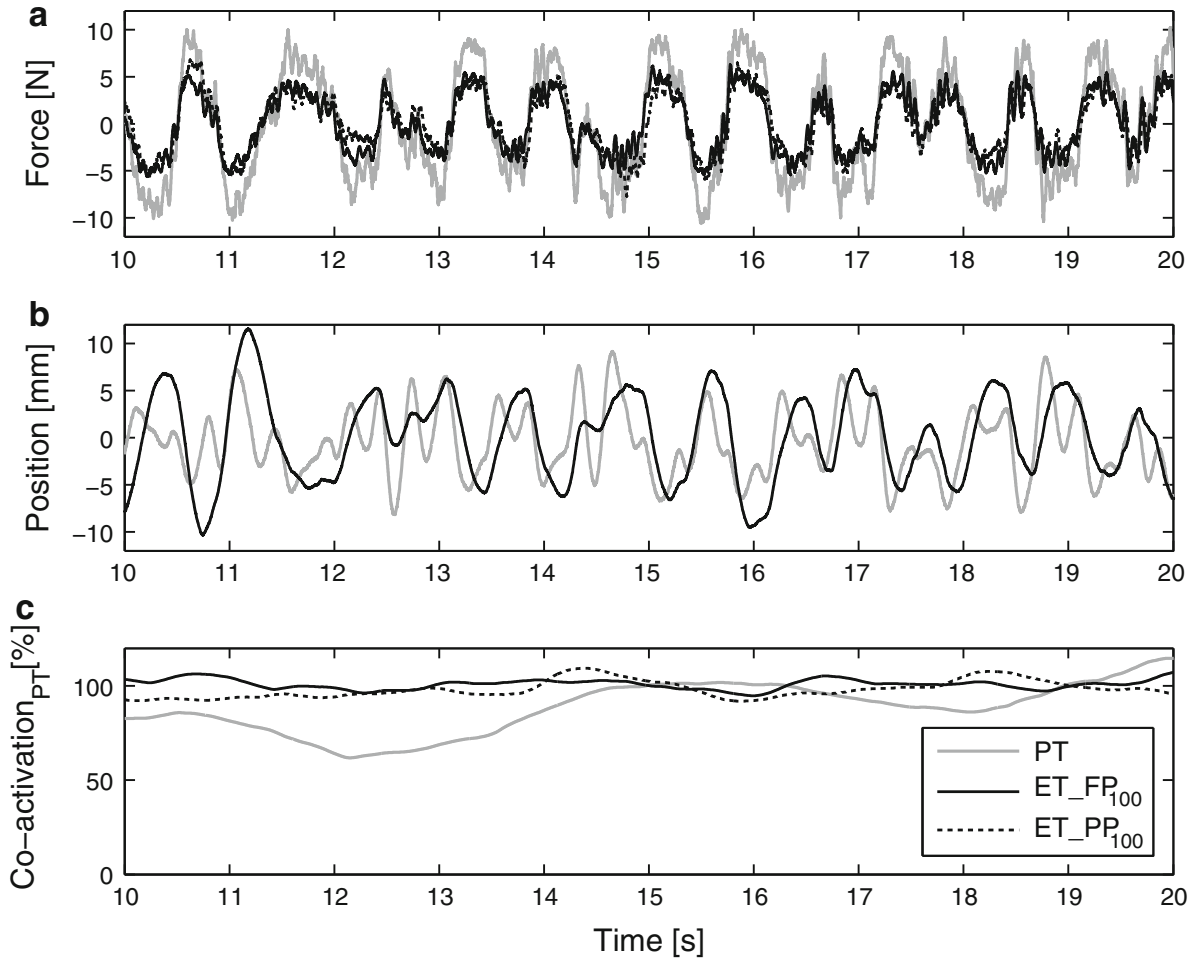

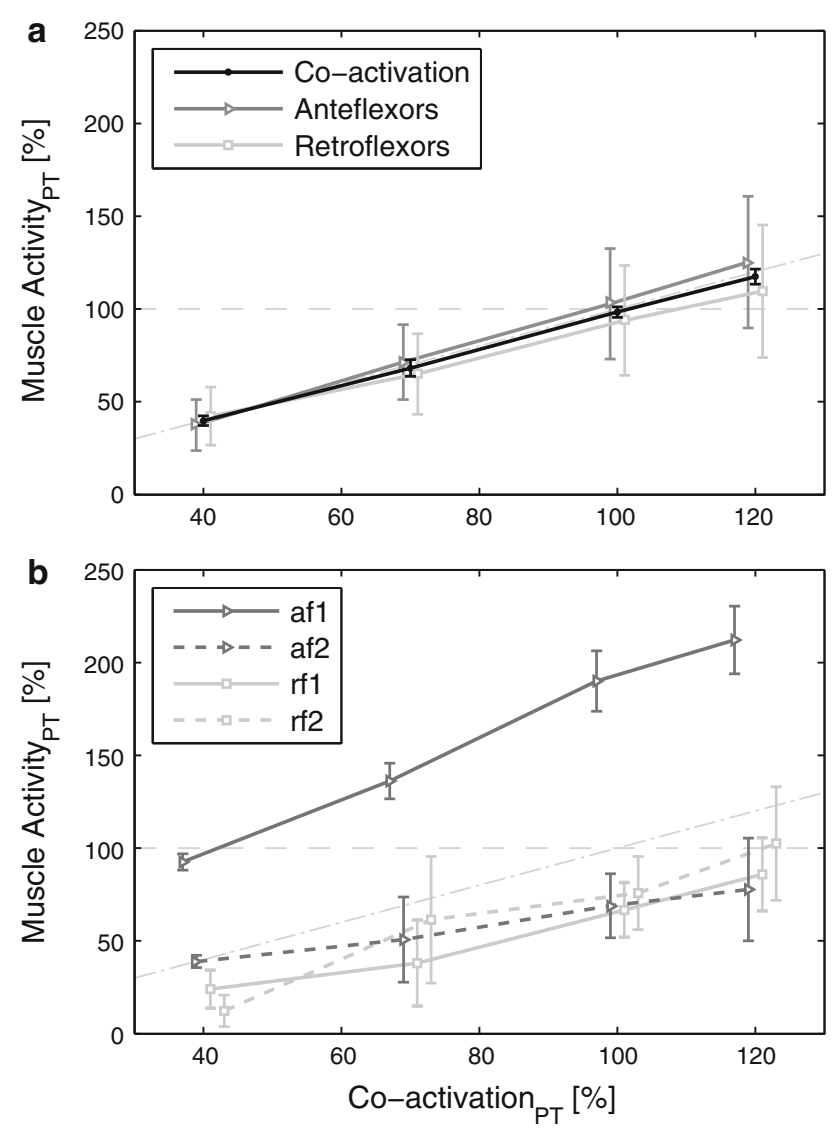

Fig. 3 Mean muscle activity signals plotted against co-activation (i.e., ET level) during ET_FP trials. In both plots, the thin dash-dotted grey line indicates where the measured signals are equal to the task condition and the thin horizontal dashed grey line represents the task level for $\mathrm{ET}_{100}$ as defined by PT. a Group averaged mean of coactivation, anteflexor and retroflexor muscle activity signals. b A signal subject mean muscle activity signals of individual muscles (af $1=\mathrm{m}$. pectoralis major, af $2=\mathrm{m}$. deltoideus anterior, $\mathrm{rf} 1=\mathrm{m}$. deltoideus posterior and $\mathrm{rf} 2=\mathrm{m}$. latissimus dorsi)

decreasing gain and $180^{\circ}$ phase lag) is dominated by the inertial properties of the arm. The intermediate frequency response (i.e., the oscillatory peak at the eigenfrequency) is due to viscous intrinsic and reflexive properties. The reflexive impedance is influenced by the velocity feedback (slope of +1 in the gain and $90^{\circ}$ phase lead for all frequencies), position feedback (flat gain at low frequencies and $0^{\circ}$ phase $)$ and a time delay $\left(0^{\circ}\right.$ at $0 \mathrm{~Hz}$ and increasing phase lag with frequency). The coherence of the mechanical admittance and reflexive impedance was similar to those reported previously for position tasks performed with this setup (Schouten et al. 2008b), thereby justifying the use of linear identification techniques.

\section{Task instruction: PT versus ET}

A substantial effect of task instruction exists in both the mechanical admittance and reflexive impedance when comparing the co-activation equivalent conditions (i.e., PT and ET_FP $\left.{ }_{100}\right)$. The mechanical admittance gain was larger, and the reflexive impedance gain was lower during ET_FP 100 . These effects were significant for the mechanical admittance $(P<0.001)$ and reflexive impedance $(P<0.05)$, both up to $1.5 \mathrm{~Hz}$. Below the eigenfrequency (approximately $4 \mathrm{~Hz}$ ), the mechanical admittance phase lag and the reflexive impedance phase lead increased during ET_FP ${ }_{100}$. These effects were significant for the mechanical admittance phase $(P<0.001)$ up to $3.0 \mathrm{~Hz}$ and the reflexive impedance phase $(P<0.05)$ up to $1.5 \mathrm{~Hz}$. All of these effects indicate decreased reflex contributions in ET_FP 100 .

Comparing ET_FP $40, \quad$ ET_FP $70, \quad$ ET_FP 100 and ET_FP 120 , higher EMG co-activation levels provided reduced admittance gains (i.e., increased impedance) and higher reflexive impedance gains (i.e., increased reflex contributions). The decreasing effect on mechanical admittance was significant $(P<0.05)$ up to $3 \mathrm{~Hz}$, and the increasing effect on reflexive impedance was significant $(P<0.05)$ up to $1.5 \mathrm{~Hz}$. Increasing EMG co-activation reduced the mechanical admittance phase lag and reflexive impedance phase lead. The decreasing effect on mechanical admittance phase lag was significant $(P<0.05)$ up to $6.4 \mathrm{~Hz}$, with the exception of the $0.5 \mathrm{~Hz}$ point which showed no significant effect. The decreasing effect on reflexive impedance lead was significant $(P<0.05)$ up to $1.5 \mathrm{~Hz}$. Notwithstanding the $0.5-\mathrm{Hz}$ point in the mechanical admittance phase response; these effects indicate a modulation of reflex activity which increased with muscle activation.

\section{Perturbation type: FP versus PP}

Figure 5 plots the mechanical admittance and reflexive impedance of a typical subject when exposed to both perturbation types during $\mathrm{ET}_{100}$ trials. Gain and phase responses were similar for FP and PP trials and no significant effect of perturbation type was found for either the mechanical admittance or the reflexive impedance at any ET level. These results match those observed in the time domain (see Fig. 2) where force was approximately equal between FP and PP, and indicate that perturbation type had no effect on the intrinsic or reflexive contributions to posture maintenance during ET trials.

Parametric identification of neuromuscular model

Given this lack of influence from the perturbation type, the neuromuscular model fitting was limited to only PT, RT and ET conditions where the FP was applied (i.e., six conditions). The optimization procedure yielded a model that accurately captured the dynamics of the system in all 

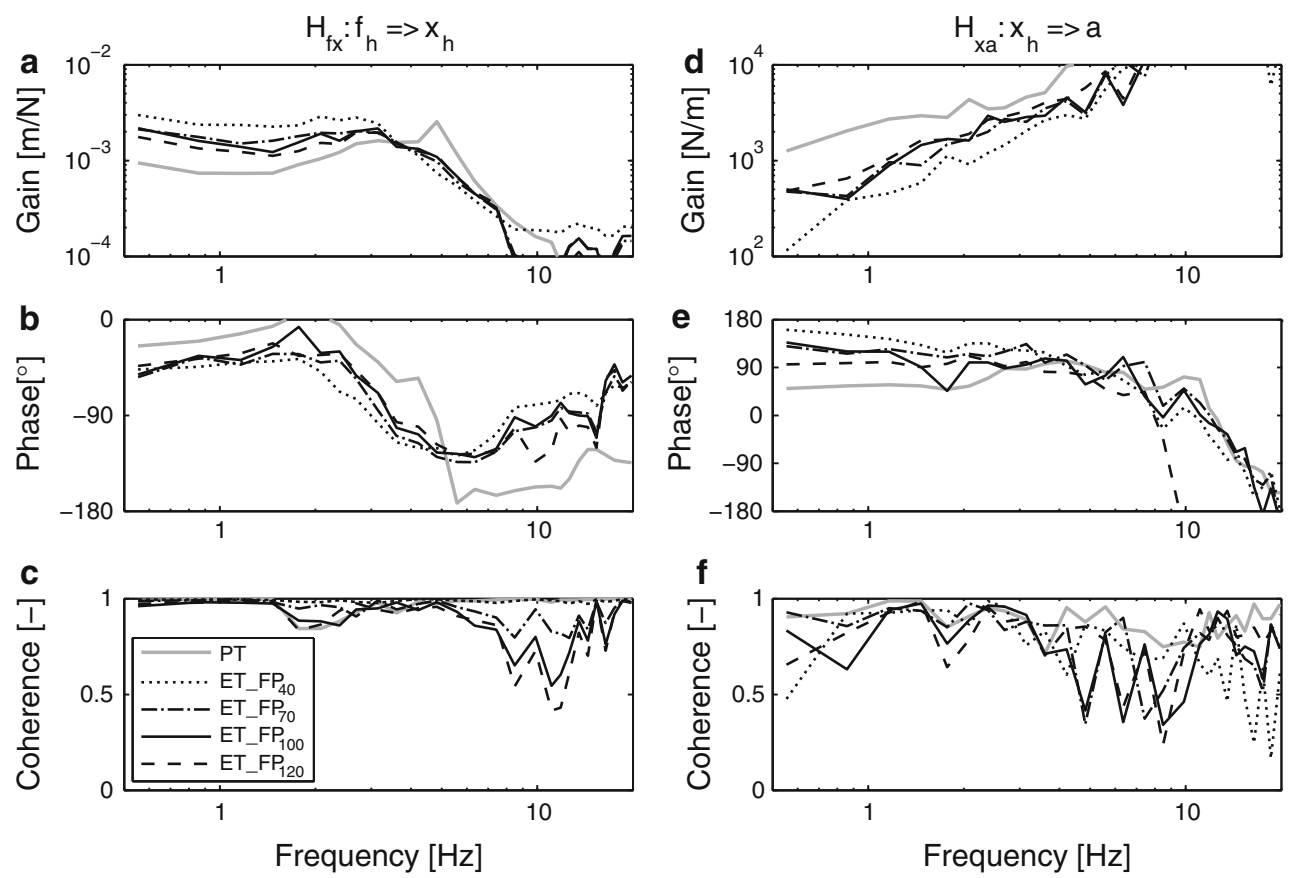

Fig. 4 FRFs of a typical subject for the mechanical admittance $(\mathbf{a}, \mathbf{b}, \mathbf{c})$ and the reflexive impedance (d, e, f) for PT (thick grey) and ET_FP (thin black) trials at the four co-activation levels (40, 70, 100 and 120). From top to bottom: magnitude, phase and coherence

conditions. The calculated $\mathrm{VAF}_{x}$ and $\mathrm{VAF}_{a}$ for all estimated conditions are provided in Table 2. The $\mathrm{VAF}_{x}$ was generally quite high (greater than $80 \%$ ), while the $\mathrm{VAF}_{a}$ was approximately $20 \%$ for all ET conditions and $30 \%$ for PT trials. The presence of substantial power at non-excited frequencies inherent within the EMG signals is reflected in the lower $\mathrm{VAF}_{a}$ values relative to $\mathrm{VAF}_{x}$. The lower $\mathrm{VAF}_{a}$ values in ET trials relative to PT trials were attributed to the lower signal-to-noise ratios associated with lower reflexive impedance magnitude. The PT results are an improvement of VAF values described in our previous studies (Schouten et al. 2008b) for similar perturbation properties (dominant power up to $1.5 \mathrm{~Hz}$ ), and this was attributed to the expanded frequency response range used in the parameter estimation as provided by the reduced power region.

Figure 6 shows the intrinsic muscular arm parameters along with the reflexive parameters for the PT and ET conditions considered in the model fitting. All estimated parameters are summarized in Table 3. The influence of task (i.e., PT vs. ET_FP 100 ) was significant for all intrinsic parameters $(P<0.05)$, which for the arm were reduced in the ET_FP 100 condition by $11 \%$ for stiffness $(k)$ and $17 \%$ for damping $(b)$. The reflexive parameters were affected to a more substantial degree, showing a significant $(P<0.001)$ influence of task condition where position feedback was effectively eliminated, velocity feedback was reduced by $36 \%$ and acceleration feedback was reduced by $39 \%$.
The influence of ET level was significant for both the intrinsic arm $(P<0.001)$ and hand grip parameters $(P<0.05)$, which as expected increased with ET level. Compared to PT, arm stiffness and damping in ET_FP 40 were 35 and $39 \%$ lower, respectively, and even in ET_FP ${ }_{120}$, arm stiffness and damping were 6 and $12 \%$ lower, respectively. This is a surprising result considering that ET_FP 120 trials were meant to produce co-contraction conditions (i.e., intrinsic stiffness and damping) 20\% higher than the PT condition. Although the intrinsic arm parameters in ET_FP 120 were on average lower than those of the PT, three of the ten subjects did produce intrinsic parameters above those obtained in PT. The influence of ET level on position feedback was not significant as it was close to zero for all ET levels. However, the ET level significantly influenced $k_{v}(P<0.001)$ and $k_{a}(P<0.05)$, both of which increased with ET level.

\section{Discussion}

Position task: comparison with previous work

A position task with force perturbations requires subjects to decrease joint admittance (i.e., increase stiffness) through the use of co-contraction and reflexes (van der Helm et al. 2002). Joint admittance is further lowered with reduced bandwidth perturbations. When a perturbation contains substantial power at frequencies below the system 

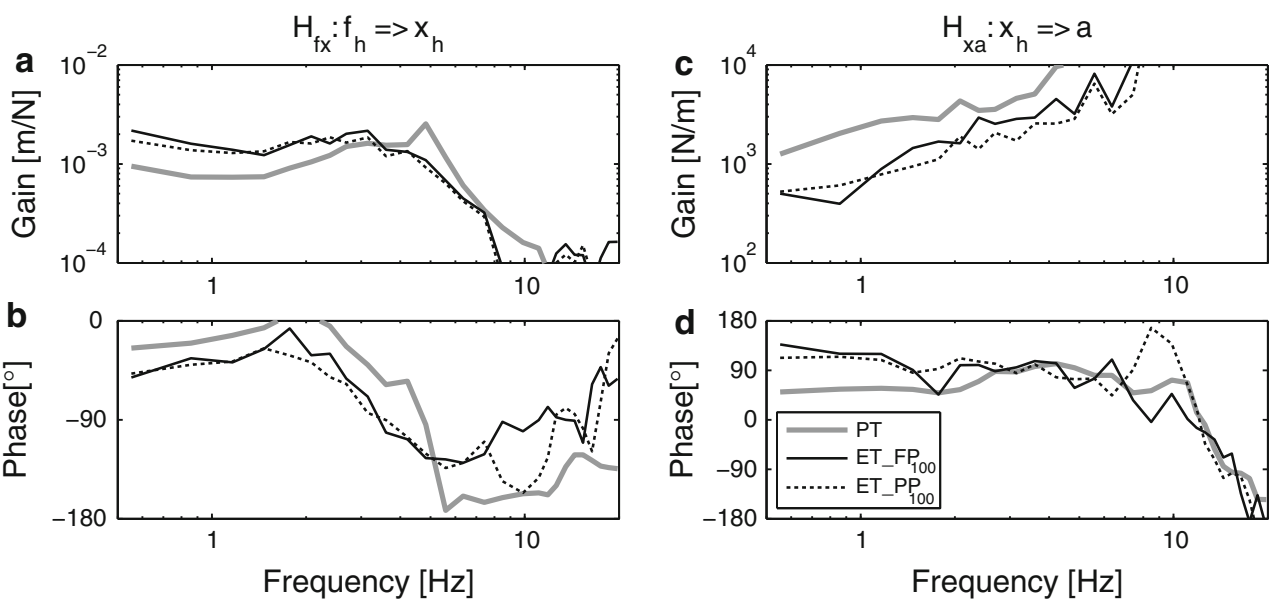

Fig. 5 FRFs of a typical subject for the mechanical admittance $(\mathbf{a}, \mathbf{b})$ and the reflexive impedance (c, d) for the $\mathrm{ET}_{100}$ trials for both force (FP) and position perturbations (PP) as well the PT trial. Top plots are magnitude and bottom plots are phase

Table 2 Validation results of neuromuscular model fits

\begin{tabular}{lll}
\hline Condition & $\mathrm{VAF}_{x}(\mathrm{SD})(\%)$ & $\mathrm{VAF}_{a}(\mathrm{SD})(\%)$ \\
\hline PT & $80.6( \pm 4.1)$ & $30.8( \pm 9.0)$ \\
RT & $91.1( \pm 8.0)$ & N/A \\
ET_FP & $90.3( \pm 3.2)$ & $19.3( \pm 16.0)$ \\
ET_FP $_{70}$ & $89.0( \pm 4.6)$ & $19.7( \pm 15.3)$ \\
ET_FP & $88.8( \pm 4.8)$ & $21.8( \pm 13.2)$ \\
ET_FP & $89.3( \pm 3.8)$ & $23.3( \pm 14.4)$ \\
\hline
\end{tabular}

eigenfrequency (in this study $0.5-1.5 \mathrm{~Hz}$ ), potential oscillations due to the neural time delay inherent with afferent feedback are avoided and task performance is improved through the use of reflexes. Including frequencies above $1.5 \mathrm{~Hz}$ with reduced power facilitates identification across the entire bandwidth of the arm while avoiding reflex suppression and reduced task performance (Mugge et al. 2007). The FRF of our PT in the low-frequency region is comparable to previous studies (van der Helm et al. 2002; Schouten et al. 2008b), and the reduced power method now highlights the effect of reflexes during posture maintenance in the form of an oscillatory peak at the eigenfrequency.

The neuromuscular parameters identified here for the shoulder are in the same order of magnitude found in previous studies from our group (van der Helm et al. 2002; Schouten et al. 2008b). The value of the time delay ( $\sim 30 \mathrm{~ms}$ ) indicates that the reflexive impedance is dominated by spinal reflexes. The PT condition elicits substantial reflex contributions to the dynamic postural control of the arm. The values of the identified reflexive parameters $k_{p}$ and $k_{v}$ are somewhat higher than those found previously. This was attributed to the inclusion of the environmental stiffness $(160 \mathrm{~N} / \mathrm{m})$, not used in previous experiments, which facilitated an inherently more stable test environment allowing subjects to make more effective use of reflexes in this postural control experiment of the arm.

Under such PT conditions, simulation studies with experimentally validated results have shown that subjects optimize performance (minimizing position variations) and energy consumption (metabolic) to produce consistent levels of static muscle activation (Schouten et al. 2001; van der Helm et al. 2002). The central nervous system weights control effort establishing reflex gain settings in order to maximize performance while maintaining stability. A different strategy seemed to be used when subjects performed ET.

Performance of the EMG task

In performing ET, subjects demonstrated the ability to easily match the mean co-activation measured in PT trials. The low variations of the co-activation signal indicated that subjects were able to make effective use of the EMG biofeedback to maximize task performance, in this case minimize variations in EMG. Subject task performance was effective across all ET levels, where the co-activation signal variation increased with ET level. This was most likely due to motor noise which is known to increase with muscular contraction effort (Hamilton et al. 2004; Tracy et al. 2005).

A limitation of this study is that the co-activation signal combines the EMG of four muscles (two agonists and two antagonists) allowing subjects to use whatever combination of muscles they perceived to be optimal to complete the task. The majority of subjects favored single muscles using a different muscle usage distribution in comparison with PT. Since no substantial drift (i.e., zero net moment) was observed in the direction of the favored muscle, it is suggested that subjects recruited unrecorded muscles-those 

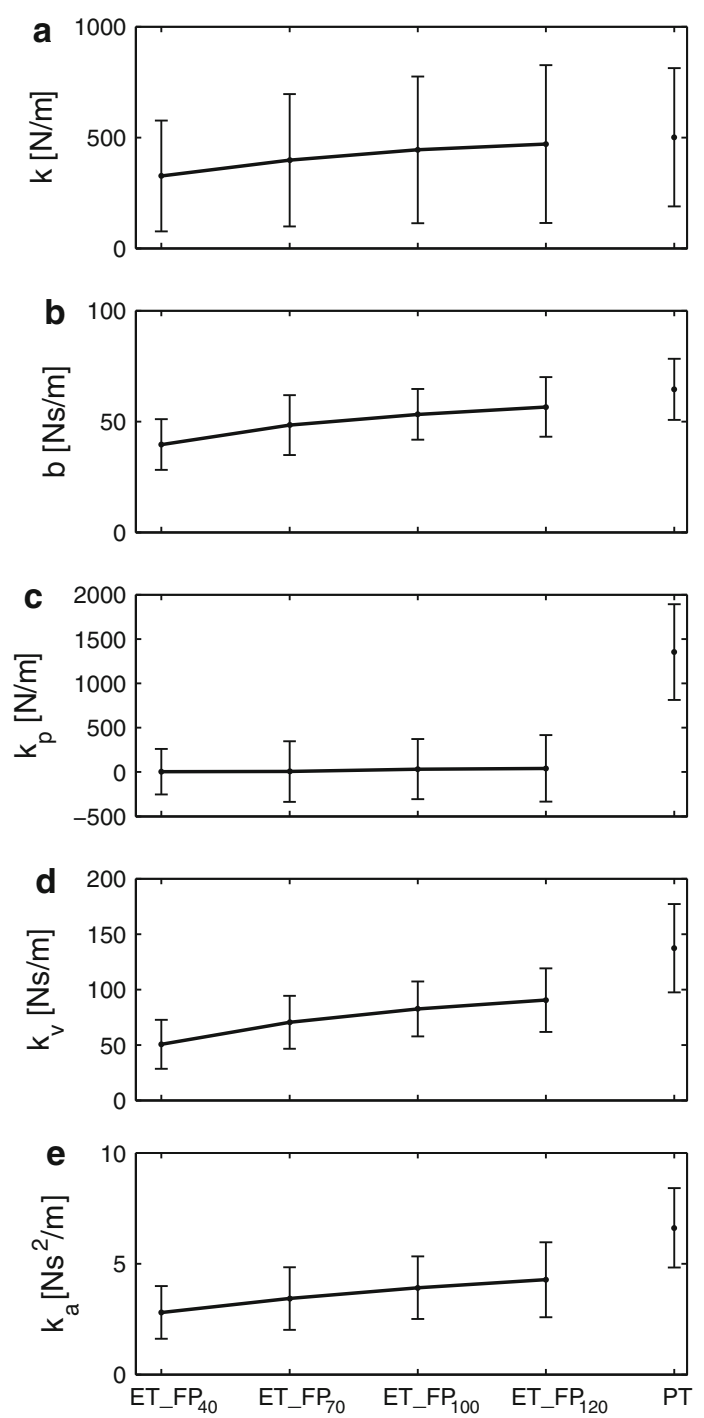

Fig. 6 Parameter estimation result of the neuromuscular control model for arm intrinsic $(\mathbf{a}, \mathbf{b})$ and reflexive parameters $(\mathbf{c}, \mathbf{d}, \mathbf{e})$. Mean and standard deviation over all subjects are shown for each parameter

which may have been less effective for posture maintenance-to compensate for the favored muscle. This would in turn result in the observed reduction in estimated intrinsic stiffness and damping parameters when comparing the co-activation equivalent PT and ET conditions. Such a response could reflect an optimal control strategy that weighs heavily toward minimizing energy consumption and control effort to complete the task. However, such a muscle recruitment strategy could not be confirmed as no additional muscles were recorded. Regardless, the muscle activation patterns chosen by subjects were consistent across ET levels and perturbation types. Therefore, these task-dependent changes do not detract from the value of these results. In fact, this task is considered a viable method to separate perturbation type from task instruction in an effort to identify the dominant factor.
Perturbation type does not affect joint dynamics

Task and perturbation type are interrelated; position tasks require a force disturbance and force tasks require a position perturbation. Previous studies (Doemges and Rack 1992; Perreault et al. 2008) identified the opposing nature of typical task-perturbation combinations (position taskforce perturbation vs. force task-position perturbations) to have a substantial effect on the reflex contribution to joint admittance. Similar results have been found when varying only task condition across consistent force (Mugge et al. 2010) and position perturbations (Dietz et al. 1994). However, to the knowledge of the authors, no previous attempts have been made to identify the effects of perturbation type across a common task instruction. The lack of significant difference in either of the estimated FRFs during ET_FP and ET_PP trials suggests that the central nervous system did not perceive any difference between the two perturbation types. These results indicate that the effect of task instruction when considering the task-perturbation link is more dominant than perturbation type in the newly developed ET.

Assuming that estimated reflex feedback gains of the ET_PP trials would yield the same results as the ET_FP trials, comparison of these ET results can be made to similar studies in the literature where constant force or EMG level tasks were used in combination with position perturbations (Kearney et al. 1997; Mirbagheri et al. 2000; Cathers et al. 2004). In those studies, it was assumed that velocity feedback plays the dominant afferent role and position feedback was negligible. Our study supports this assumption by estimating position feedback to be zero during our ET trials. However, this further indicates that constant force or EMG level matching tasks with position perturbations represents an experimental condition which does not elicit a functional use of reflexes. Instead, it is suggested that force perturbations with position tasks provide a more appropriate experimental paradigm to investigate reflex contributions to dynamic motor control of the arm.

Position task enhances afferent feedback

Modulation of reflex rather than intrinsic parameters more substantially affected the observed changes in the FRFs (increasing admittance and phase lag, and decreasing resonant frequency and peak) when comparing PT and ET trials. In particular, position feedback was effectively eliminated in the ET_FP 100 trials, while velocity and acceleration feedback dropped 36 and 39\%, respectively, relative to PT. Although it could be argued that the suppression of reflexes aligns with an optimal task performance to minimize the variation of the co-activation signal 
Table 3 Parameter estimation results of all intrinsic and reflexive parameters; mean and standard deviation (Mean \pm SD) over all subjects $(n=10)$ are shown for each parameter

\begin{tabular}{|c|c|c|c|c|c|c|}
\hline Parameters & PT & RT & ET_FP 40 & $\mathrm{ET}_{-} \mathrm{FP}_{70}$ & $\mathrm{ET}_{-} \mathrm{FP}_{100}$ & ET_FP $_{120}$ \\
\hline$t_{d}(\mathrm{~s})$ & $0.030 \pm 0.004$ & & & & & \\
\hline$m(\mathrm{~kg})$ & $2.23 \pm 0.39$ & & & & & \\
\hline$k(\mathrm{~N} / \mathrm{m})$ & $506 \pm 305$ & $177 \pm 40$ & $330 \pm 247$ & $401 \pm 294$ & $448 \pm 327$ & $475 \pm 351$ \\
\hline$b(\mathrm{Ns} / \mathrm{m})$ & $64 \pm 14$ & $13 \pm 4$ & $40 \pm 11$ & $48 \pm 48$ & $53 \pm 11$ & $57 \pm 13$ \\
\hline$k_{h}(\mathrm{kN} / \mathrm{m})$ & $31 \pm 12$ & $5 \pm 3$ & $10 \pm 9$ & $12 \pm 11$ & $14 \pm 10$ & $16 \pm 14$ \\
\hline$b_{h}(\mathrm{Ns} / \mathrm{m})$ & $339 \pm 151$ & $75 \pm 39$ & $100 \pm 91$ & $127 \pm 112$ & $143 \pm 114$ & $165 \pm 151$ \\
\hline$k_{p}(\mathrm{~N} / \mathrm{m})$ & $1,348 \pm 540$ & - & $3 \pm 257$ & $6 \pm 342$ & $33 \pm 339$ & $41 \pm 376$ \\
\hline$k_{v}(\mathrm{Ns} / \mathrm{m})$ & $137 \pm 40$ & - & $51 \pm 22$ & $70 \pm 24$ & $82 \pm 25$ & $80 \pm 29$ \\
\hline$k_{a}\left(\mathrm{Ns}^{2} / \mathrm{m}\right)$ & $6.6 \pm 1.8$ & - & $2.8 \pm 1.2$ & $3.4 \pm 1.4$ & $3.9 \pm 1.4$ & $4.3 \pm 1.7$ \\
\hline
\end{tabular}

and maintain a constant level, the low-pass filtering of the displayed co-activation signal at a cutoff of $0.3 \mathrm{~Hz}$ was meant to avoid this suppression. The presence of velocity feedback, which in ET_FP 100 was greater than the intrinsic damping, suggests this was achieved. This combined with the minor increase $(\sim 4 \%)$ in the co-activation signal standard deviation observed when perturbations were applied suggests that the co-activation signal produced by subjects truly reflected a state of intrinsic muscular cocontraction.

From a control engineering perspective, the enhanced spindle reflexes during PT (position feedback in particular) are analogous to a position servo, where position feedback is required for optimal performance. In this study, this contribution is significantly enhanced by employing low bandwidth perturbations. On the other hand, during ET (or similarly force tasks) where a steady EMG is preferred, position feedback would only add to the deviations reducing task performance. As a result, subjects actively suppress position feedback during such tasks.

The separate modulation of reflex gains may be possible via two physiological mechanisms: presynaptic modulation or $\gamma$-motor neuron modulation. Presynaptic modulation is facilitated by interneurons which inhibit or excite Ia afferents (Stein and Capaday 1988). However, as the Ia afferent contains primarily stretch velocity information and the velocity feedback was shown to decrease only moderately, this effect is unlikely to have facilitated the position feedback modulation. On the other hand, $\gamma$-motor neurons activate the intrafusal muscle fibers using separate static (i.e., position) and dynamic (i.e., velocity) pathways (Hulliger et al. 1989). The separate reflex modulation of the position and velocity feedback may therefore have been responsible for responses seen in this study. Similar arguments have been made in studies by Ludvig et al. (2007) and Ludvig and Kearney (2007) where through a unique experimental paradigm, subjects were able to voluntarily modulate their reflexes in real time.
The reflex modulation observed with increasing ET levels is similar to the findings of many others (Neilson 1972; Matthews 1986; Kirsch et al. 1993; Cathers et al. 2004; Schouten et al. 2008b), in both the FRFs and the reflex gains. Position feedback remained constant and close to zero across all ET levels for each subject, indicating a task-dependent suppression of position feedback without dependence on activation level. The increasing velocity feedback gain on the other hand represents a muscle activation appropriate reflex response (Matthews 1986; Cathers et al. 2004). This study represents the first attempt to separate the position and velocity feedback contributions at different levels of muscle activation and helps explain and confirm the variation seen in many other studies of a similar experimental paradigm as being primarily due to velocity feedback.

\section{Conclusions}

The experiments demonstrate a postural control paradigm of the arm with reflexive and intrinsic stiffness contributions that depend heavily on the task instruction and not on the perturbation type. Position tasks enhance joint impedance and afferent feedback, compared to EMG tasks, which through neuromuscular model fitting was found to result primarily from decreased afferent contributions. Muscle visco-elasticity decreased by approximately $14 \%$ in coactivation matching ET trials, possibly due to a different muscle recruitment strategy to minimize energy consumption. Afferent position feedback was suppressed in all ET trials and velocity and acceleration feedback decreased by approximately $37 \%$ in co-activation matching ET trials. Concluding, this study indicates that force perturbations with position tasks are a more effective paradigm to study complete dynamic motor control of the arm, while EMG tasks, regardless of perturbation type, tend to reduce the afferent contribution. 
Acknowledgments This research is supported by the Dutch Technology Foundation STW, which is part of the Netherlands Organization for Scientific Research (NWO) and partly funded by the Ministry of Economic Affairs, Agriculture and Innovation.

Open Access This article is distributed under the terms of the Creative Commons Attribution Noncommercial License which permits any noncommercial use, distribution, and reproduction in any medium, provided the original author(s) and source are credited.

\section{Appendix 1: muscle activation signal}

The muscle activation signal $a(t)$ estimates the net amount of force generated using the EMG signals and is used in the non-parametric system identification. A prewhitening filter was applied to the raw measurements to improve the quality of the signals following the procedures of Clancy et al. (2002). The parameters for the filter (sixth order) were obtained from the power spectral densities of the $25 \mathrm{~N}$ isometric push and pull task. The results of all isometric push-pull tasks were then used to estimate the force-to-EMG relationship by linear regression. The total muscle activation was then obtained by summing the scaled rectified prewhitened EMGs of the four muscles according to:

$a(t)=\frac{1}{2} \sum_{i=1}^{4} K_{i}\left|e_{w, i}(t)\right|$

where $K_{i}$ is the EMG-to-force scale factors for each muscle, being positive for the two anteflexors and negative for the two retroflexors. This equation assumes that the two muscles generating either push or pull have equal relevance and that their mean value represents the total force being generated in that direction.

\section{Appendix 2: model description}

Figure 7 shows the entire model structure including the external force disturbance, environment and arm. The external force disturbance $D(s)$ minus the force measured at the handle $F_{h}(s)$ was the input for the environment.

The environment was modeled as a second-order massspring-damper system which did not vary between conditions

$H_{e}(s)=\frac{1}{m_{e} s^{2}+b_{e} s+k_{e}}$

where $s=j 2 \pi f$. The arm model $H_{\text {arm }}(s)$ included the intrinsic parameters captured in $H_{\text {int }}(s)$, representing the mass as well as the muscle viscoelasticity which varied per EMG task condition

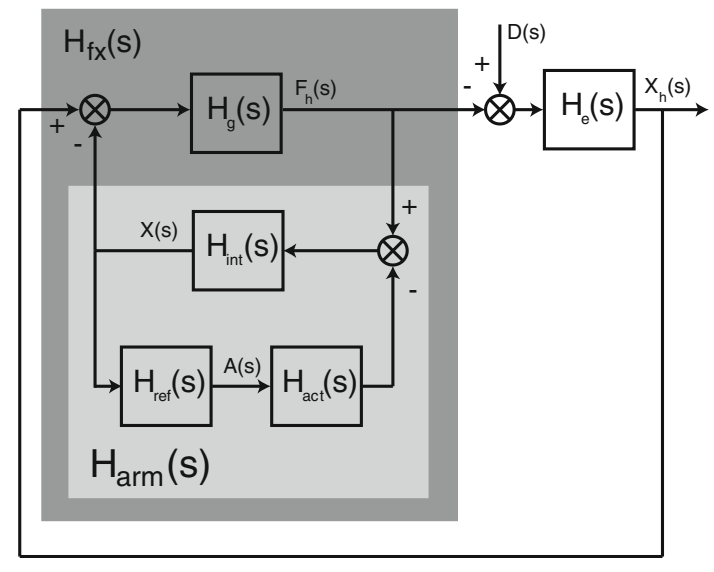

Fig. 7 NMS model $H_{f x}(s)$ in conjunction with the environmental model $H_{e}(s)$. The external force disturbance $D(s)$, hand force $F(s)$, position of the handle $X(s)$, and muscle activation $A(s)$ are measured. $H_{g}(s)$ represents grip dynamics, $H_{\text {int }}(s)$ intrinsic properties, $H_{\text {act }}(s)$ activation dynamics, $H_{\text {ref }}(s)$ reflexive feedback and $X(s)$ position of the arm. The light grey box $H_{\text {arm }}(s)$ represents the arm model without grip

$H_{\text {int }}(s)=\frac{1}{m s^{2}+b s+k}$

The reflexive response of the arm model $H_{\text {ref }}(s)$ was described using muscle spindle position $\left(k_{p}\right)$, velocity $\left(k_{v}\right)$ and acceleration $\left(k_{a}\right)$ feedback terms in series with a neural signal transmission time delay $\left(\tau_{d}\right)$

$H_{\text {ref }}(s)=\left(k_{a} s^{2}+k_{v} s+k_{p}\right) e^{\left(-\tau_{d} s\right)}$

Muscle activation dynamics were modeled as a secondorder system, with eigenfrequency $\left(f_{0}=\omega_{0} / 2 \pi\right)$ and relative damping $(\beta)$. These parameters were obtained from previous estimates made in a study by Schouten et al. $2008 b$

$H_{\text {act }}(s)=\frac{1}{\frac{1}{\omega_{0}^{2}} s^{2}+\frac{2 \beta}{\omega_{0}} s+1}$

The hand grip was modeled between the environment and the arm with dynamics described as a spring and damper

$H_{g}(s)=b_{h} s+k_{h}$

Combining the above equations provided the modeled description of $H_{\text {arm }}(s)$, which in turn is used to describe the mechanical admittance and reflexive impedance as

$H_{\text {arm }}(s)=\frac{H_{\text {int }}(s)}{1+H_{\text {int }}(s) H_{\text {ref }}(s) H_{\text {act }}(s)}$
$H_{f x}(s)=\frac{X_{h}(s)}{F_{h}(s)}=H_{\text {arm }}(s)+H_{g}^{-1}(s)$ 
$H_{a x}(s)=\frac{A(s)}{X_{h}(s)}=H_{\text {ref }}(s) \frac{H_{g}(s)}{H_{g}(s)+H_{\text {arm }}^{-1}(s)}$

Note that because force and displacement could only be measured after the handle, the arm admittance and reflexive impedance includes the hand grip dynamics. As the grip stiffness and damping increase $H_{f_{x}}(s)$ becomes $H_{\text {arm }}(s)$ and $H_{\text {ax }}(s)$ becomes $H_{\text {ref }}(s)$.

\section{References}

Agarwal GC, Gottlieb GL (1977) Compliance of the human ankle joint. J Biomech Eng 99:166-170

Akazawa K, Milner TE, Stein RB (1983) Modulation of reflex EMG and stiffness in response to stretch of human finger muscle. J Neurophysiol 49:16-27

Basmajian JV (1981) Biofeedback in rehabilitation-a review of principles and practices. Arch Phys Med Rehabil 62:469-475

Cathers I, O'Dwyer N, Neilson P (1999) Dependence of stretch reflexes on amplitude and bandwidth of stretch in human wrist muscle. Exp Brain Res 129:278-287

Cathers I, O'Dwyer N, Neilson P (2004) Variation of magnitude and timing of wrist flexor stretch reflex across the full range of voluntary activation. Exp Brain Res 157:324-335

Clancy EA, Morin EL, Merletti R (2002) Sampling, noise-reduction and amplitude estimation issues in surface electromyography. J Electromyogr Kinesiol 12:1-16

de Vlugt E, Schouten AC, van der Helm FCT (2002) Adaptation of reflexive feedback during arm posture to different environments. Biol Cybern 87:10-26

Dietz V, Discher M, Trippel M (1994) Task-dependent modulation of short-latency and long-latency electromyographic responses in upper-limb muscles. Electroencephalogr Clin Neurophysiol 93:49-56

Doemges F, Rack PMH (1992) Task-dependent changes in the response of human wrist joints to mechanical disturbance. J Physiol Lond 447:575-585

Hamilton AFD, Jones KE, Wolpert DM (2004) The scaling of motor noise with muscle strength and motor unit number in humans. Exp Brain Res 157:417-430

Holtermann A, Mork PJ, Andersen LL, Olsen HB, Sogaard K (2010) The use of EMG biofeedback for learning of selective activation of intra-muscular parts within the serratus anterior muscle: a novel approach for rehabilitation of scapular muscle imbalance. J Electromyogr Kinesiol 20:359-365

Hulliger M, Durmuller N, Prochazka A, Trend P (1989) Flexible fusimotor control of muscle-spindle feedback during a variety of natural movements. Prog Brain Res 80:87-101

Jenkins G, Watts D (1968) Spectral analysis and its applications. Holden-Day, San Francisco

Kearney RE, Hunter IW (1982) Dynamics of human ankle stiffnessvariation with displacement amplitude. J Biomech 15:753-756

Kearney RE, Stein RB, Parameswaran L (1997) Identification of intrinsic and reflex contributions to human ankle stiffness dynamics. IEEE Trans Biomed Eng 44:493-504

Kirsch RF, Kearney RE, Macneil JB (1993) Identification of timevarying dynamics of the human triceps surae stretch reflex. 1 . Rapid isometric contraction. Exp Brain Res 97:115-127

Kukreja SL, Galiana HL, Kearney RE (2003) NARMAX representation and identification of ankle dynamics. IEEE Trans Biomed Eng 50:70-81
Ludvig D, Kearney RE (2007) Real-time estimation of intrinsic and reflex stiffness. IEEE Trans Biomed Eng 54:1875-1884

Ludvig D, Cathers I, Kearney RE (2007) Voluntary modulation of human stretch reflexes. Exp Brain Res 183:201-213

Matthews PBC (1986) Observations on the automatic compensation of reflex gain on varying the preexisting level of motor discharge in man. J Physiol Lond 374:73-90

Mirbagheri MM, Barbeau H, Kearney RE (2000) Intrinsic and reflex contributions to human ankle stiffness: variation with activation level and position. Exp Brain Res 135:423-436

Mugge W, Abbink DA, van der Helm FCT (2007) Reduced power method: how to evoke low-bandwidth behaviour while estimating full-bandwidth dynamics. In: IEEE 10th International conference on rehabilitation robotics, vols 1 and 2. IEEE, New York, pp 575-581

Mugge W, Abbink DA, Schouten AC, Dewald JPA, van der Helm FCT (2010) A rigorous model of reflex function indicates that position and force feedback are flexibly tuned to position and force tasks. Exp Brain Res 200:325-340

Neilson PD (1972) Frequency-response characteristics of tonic stretch reflexes of biceps brachii muscle of intact man. Med Biol Eng 10:460

Perreault EJ, Chen K, Trumbower RD, Lewis G (2008) Interactions with compliant loads alter stretch reflex gains but not intermuscular coordination. J Neurophysiol 99:2101-2113

Pintelon R, Schoukens J (2001) System identification: a frequency domain approach. IEEE Press, New York

Ruitenbeek JC, Janssen RJ (1984) Computer-controlled manipulator display system for human-movement science. Med Biol Eng Comput 22:304-308

Schouten AC, de Vlugt E, van der Helm FCT, Brouwn GG (2001) Optimal posture control of a musculo-skeletal arm model. Biol Cybern 84:143-152

Schouten AC, de Vlugt E, van der Helm FCT (2008a) Design of perturbation signals for the estimation of proprioceptive reflexes. IEEE Trans Biomed Eng 55:1612-1619

Schouten AC, de Vlugt E, van Hilten JJB, van der Helm FCT (2008b) Quantifying proprioceptive reflexes during position control of the human arm. IEEE Trans Biomed Eng 55:311-321

Sinkjaer T, Toft E, Andreassen S, Hornemann BC (1988) Musclestiffness in human ankle dorsiflexors-intrinsic and reflex contributions. J Neurophysiol 60:1110-1121

Stein RB, Capaday C (1988) The modulation of human reflexes during functional motor-tasks. Trends Neurosci 11:328-332

Stein RB, Kearney RE (1995) Nonlinear behavior of muscle reflexes at the human ankle joint. J Neurophysiol 73:65-72

Stienen AHA, Schouten AC, Schuurmans J, van der Helm FCT (2007) Analysis of reflex modulation with a biologically realistic neural network. J Comput Neurosci 23:333-348

Tracy BL, Maluf KS, Stephenson JL, Hunter SK, Enoka RM (2005) Variability of motor unit discharge and force fluctuations across a range of muscle forces in older adults. Muscle Nerve 32:533-540

van der Helm FCT, Schouten AC, de Vlugt E, Brouwn GG (2002) Identification of intrinsic and reflexive components of human arm dynamics during postural control. J Neurosci Methods 119:1-14

Wu G, van der Helm FCT, Veeger HEJ, Makhsous M, Van Roy P, Anglin C, Nagels J, Karduna AR, McQuade K, Wang XG, Werner FW, Buchholz B (2005) ISB recommendation on definitions of joint coordinate systems of various joints for the reporting of human joint motion-part II: shoulder, elbow, wrist and hand. J Biomech 38:981-992 